EPJ Web of Conferences 97,00029 (2015)

DOI: $10.1051 /$ epjconf/ 20159700029

(C) Owned by the authors, published by EDP Sciences, 2015

\title{
Study of hyperon-pion resonances from kaonic absorption with KLOE
}

\author{
Oton Vázquez Doce ${ }^{1, a}$ \\ ${ }^{1}$ Excellence Cluster Universe, Technische Universität München, Garching, Germany
}

\begin{abstract}
.
Through the study of the absorptions of low momentum kaons in light target $\left({ }^{4} \mathrm{He}\right.$ and ${ }^{12} \mathrm{C}$ ), aspects of the hyperon-nucleon interaction in medium are investigated with the KLOE drift chamber with almost full acceptance.

The in-medium modification of hadron properties is the main field of study for the strangeness sector in the non-perturbative regime of the low energy region of QCD. The behaviour of strange hadrons at extreme densities are of capital importance for the description of the nuclear equation of state and the evaluation of the strangeness component in the core of the neutron stars.

The study of the antiK-hadron interactions inside the drift chamber of KLOE was initiated in order to search for signals from the formation of deeply bound kaonic nuclear states and the study of resonances like the $\Lambda(1405)$ and the $\Sigma(1385)$, and constitute a first step towards the preparation of the AMADEUS experiment at DAFNE, the $\mathrm{e}^{+} \mathrm{e}^{-}$collider of the Frascati National Laboratories (Italy) of INFN.
\end{abstract}

\section{Introduction}

The KLOE spectrometer [1] consists of a cylindrical drift chamber (DC) filled with a gas mixture of $90 \%{ }^{4} \mathrm{He}$ and $10 \%$ isobutane $\left(\mathrm{C}_{4} \mathrm{H}_{10}\right)$ surrounded by an electromagnetic calorimeter (EMC). The DAFNE accelerator provides an almost monochromatic beam of kaons $(\sim 127 \mathrm{MeV} / \mathrm{c})$. A sample of $1.4 \mathrm{fb}^{-1}$ collected during the 2004-2005 campaigns has been analyzed and output distributions for invariant masses, missing masses, momenta, etc., are available for different channels $(\Sigma \pi, \Lambda \mathrm{p}, \Lambda \pi)$ with almost full acceptance. These events correspond to the absorptions of negative kaons (stopped or in-flight) in the entrance wall of the DC, made of $400 \mu \mathrm{m}$ of carbon fiber, and in the gas filling the chamber.

The initial motivation for the investigations of the $K^{-}$absorptions inside the KLOE drift chamber was to search for the formation of deeply bound kaonic nuclear states [2]. This kind of kaonic clusters would be formed when a $K^{-}$is attached to 2 or more nucleons, and its binding energy and width could be determined studying their decay into baryons and nucleons. In particular the dibarionic state $K^{-} p p$ is expected to decay into $\Lambda p$.

Different predictions for the strenght of the antikaon-nucleon potential exist, leading to different values for the binding energies of the $K^{-}$nuclei. From an experimental point of view, two main

\footnotetext{
ae-mail: oton.vazquez@universe-cluster.de
} 
approaches have been used for studying the $K^{-} p p$ cluster: $p-p$ and heavy ion collisions [3] [4], and low momentum or stopped $K^{-}$interactions in light nuclei [5] [6]. The interpretation of the later is far from being conclusive, and it requires an accurate description of the single and multi-nucleon absoption processes that a $K^{-}$would undergo when interacting with light nuclei.

The formation of strange resonances following kaonic absorptions can be as well investigated, in particular the $\Sigma(1385)$ and the very debated $\Lambda(1405)$, whose nature still remains unsettled. The three quark picture (uds) has some difficulties in explaining the observed $\Lambda(1405)$ mass. In the context of chiral unitary models [7] two poles emerge in the scattering amplitude (with $S=-1$ and $I=0$ in the neighborhood of the $\Lambda(1405)$ mass, both contributing to the final experimental invariant mass distribution, the lower pole being dominantly coupled to the $\Sigma \pi$ channel [8]. What experimentally has been obtained from absorption experiments (in bubble chamber [9] or emulsion [10]) refers only to the charged decay channels, that contains the contribution by the $\Sigma(1385)$ resonance too, with spectra always cut at the at-rest limit, which in the KLOE case will be complemented with the in-flight absorptions at a momentum of around $120 \mathrm{MeV} / \mathrm{c}$. Non-absorption events present spectra in the $\Sigma^{0} \pi^{0}$ channel from kaon [11] and pion beams [12], and lately with photo production [13] and pp collisions [14] resulting in different line shapes.

\section{The $\Sigma(1385)$ and the $\Lambda(1405)$ in the KLOE data}

The process $\mathrm{K}^{-4} \mathrm{He} \rightarrow \Lambda \pi^{-3} \mathrm{He}$ has been investigated for absorptions in the DC gas volume, in order to extract the resonant contribution of proccesses occurring through the intermediate formation of a $\Sigma^{-}(1385)$.

The detection of a $\Lambda$ hyperon through its decay into a proton and a negative pion (with a branching ratio of 64\%) was used for tagging. The proton and pion tracks are identified with the DC and its momentum is measured with a precision better than $1 \%$ (in the case of the proton). The decay vertex is known with an error below $1 \mathrm{~mm}$. For decays inside the gas volume, the Lambda invariant mass is obtained with a resolution of $0.3 \mathrm{MeV} / \mathrm{c}^{2}$ and a value of $1115.723 \pm 0.003 \mathrm{MeV} / \mathrm{c}^{2}$ (error only statistical).

Assuming a known lineshape for the resonance, the calculation of the absorption rate is obtained assuming three-body interaction, involving a P-wave intermediate $\Sigma(1385)$ resonance or a S-wave non-resonant production. The resonant production is allowed, in the S-state of the atom, by the three body structure of the system (kaon, neutron, residual), when the $K^{-} n$ state is P-wave and correspondingly the residual is in a $\mathrm{P}$-state with respect to the $K^{-} n$ system, as follows from the angular momentum conservation.

The extraction of the ratio between the two processes is obtained with a multi-fit to the relevant kinematical variables (invariant mass, momentum and angular correlation between $\Lambda$ and $\pi$ ), both for in-flight and at rest absorptions), and preliminary results gives a ratio of resonant/non-resonant of 1.26 \pm 0.06 (stat).

For the study of the $\Lambda(1405)$, the $\Sigma^{0} \pi^{0}$ decay channel has been analyzed. The selection of events proceeds after the identification of a $\Lambda$, with the identification of three photon clusters via the EMC. Three calorimeter hits not associated to any DC track, and in time with the lambda decay vertex, are searched for and selected on the base of a $\chi^{2}$ for the TOF and the reconstructed $\pi^{0}$ mass.

The obtained invariant mass for $\Sigma^{0} \pi^{0}$ is shown in the left part of Fig. 1 for events selected in ${ }^{12} \mathrm{C}$ (DC wall). The mass distribution shows an excess of events above the kinematical limit for $\mathrm{K}^{-}$ absorptions at rest. The kinematical limit is almost the same for absorptions at rest in carbon and helium ( $1416 \mathrm{MeV} / \mathrm{c}^{2}$ in ${ }^{12} \mathrm{C}$ and $1412 \mathrm{MeV} / \mathrm{c}^{2}$ in ${ }^{4} \mathrm{He}$ ) as it depends on the last nucleon binding energy which differs only by $4 \mathrm{MeV}$ for the two nuclei. Based on this, the mass spectra was split into in-flight and at rest candidate events. 


\section{$3 \Lambda$-proton correlations}

The invariant mass from the $\Lambda$ p inclusive analysis can be seen in the right part of Fig. 1 for the DC gas volume events. A prominent enhancement is seen in the lower energy region. This part of the spectrum is expected to be populated mainly by events coming from the single nucleon absorption process, where the kaon is absorbed by a single nucleon, and a pair hyperon-pion is produced, the proton in the final state coming from a $\Sigma / \Lambda$ nuclear conversion process [15] that can take place in the residual nucleus. As explained before, the knowledge of the shape of the $\Lambda \mathrm{p}$ invariant mass for the single and multi-nucleon absorption processes is of great importance in the search for the kaonic bound clusters.

The lower invariant mass region can in fact be explored in more detail. A common vertex for the $\Lambda \mathrm{p}$ and an additional negative pion is found in approximately $1 / 6$ of the $\Lambda \mathrm{p}$ events, and the invariant mass for this subsample is represented by the dashed line in Fig. 1 (right), with an arbitrary normalization, in order to be compared with the full spectrum. With the requirement of the presence of a pion track, the contribution from the high energy tail loses strength, when compared with the inclusive selection and a cusp effect is seen around the $\Sigma \mathrm{N}$ mass threshold.
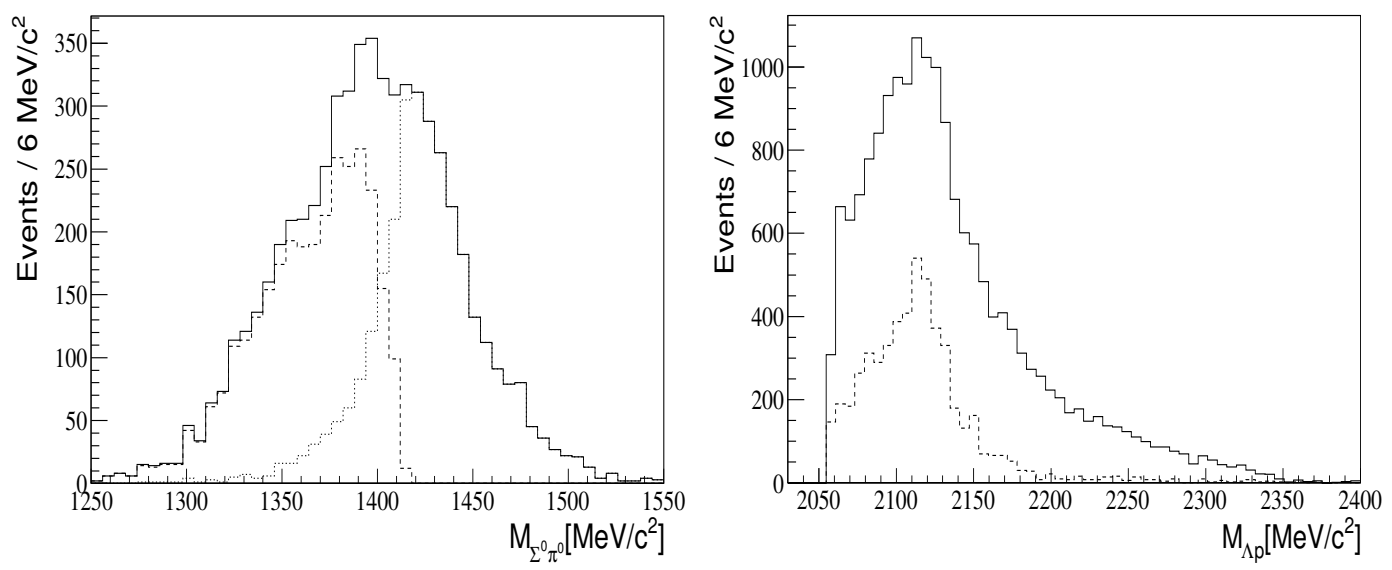

Figure 1. Right: $\Sigma^{0} \pi^{0}$ invariant mass (dashed and pointed line represent the at-rest and in-flight components respectively). Left: $\Lambda \mathrm{p}$ invariant mass for the inclusive $\Lambda \mathrm{p}$ selection (continuous line) and for events with an extra $\pi^{-}$detected (dashed line).

\section{Conclusions}

The significance of the $\mathrm{P}$-wave interaction in the $K N$ system is established with the evaluation of the resonant component in the $\mathrm{K}^{-4} \mathrm{He} \rightarrow \Lambda \pi^{-3} \mathrm{He}$. For an analogous analysis of the $\Lambda(1405)$ it is very important to achieve a clear distinction between the in-flight and at-rest spectral shape of the $\Lambda(1405)$ to correctly interpret the results and compare the last to theoretical models, with a characterization of the line shapes of the resonance. The specific theoretical predictions for the mass distributions of the $\Lambda(1405)$ can be directly compared with the results obtained and the two pole version of the theories.

From the lambda-baryon channel analyses, the determination of the fraction single/multi nucleon absorption in ${ }^{4} \mathrm{He}$ and ${ }^{12} \mathrm{C}$ will be obtained, as well as the rates of nuclear $\Sigma / \Lambda$ conversion in for the 
given energy range. The results can give strength to models in which the interaction takes place with a free proton or to those with a bound proton, and can be related to the sub threshold modification of the anti-KN interaction.

The interpretation of the data obtained with KLOE will allow to obtain a complete and firm result, with qualitative and quantitative outputs from $\mathrm{K}^{-}$absorption to be added to the pp and heavy ion collisions data.

\section{References}

[1] M. Adinolfi et al., Nucl. Instr. Meth. A 488, 51-73 (2002).

[2] Y. Akaishi and T. Yamazaki, Nucl. Phys. A 792, 229 (2007).

[3] M. Maggiora et al., Nucl. Phys. A 835, 43-50 (2010).

[4] G. Agakishiev et al., arXiv:1410.8188, nucl-ex (2014).

[5] M. Maggiora et al., Nucl. Phys. A 835, 43-50 (2010).

[6] M. Agnello et al., Phys. Rev. Lett. 94, 919303 (2005)

[7] E. Oset, A. Ramos, Nuclear Phys. A 635, 99 (1998).

[8] Y. Ikeda, T. Hyodo, W. Weise, Nucl. Phys. A 881, 98 (2012).

[9] D. Riley et al., Phys. Rev. D 11, 3065 (1975).

[10] P. J. Carlson et al., Nuclear Phys. 74, 642 (1965).

[11] Prakhov, et al., Phys. Rev. C 70, 034605 (2004).

[12] D. W. Thomas, A. Engler, H. E. Fisk, and R. W. Kraemer, Nucl. Phys. B 56, 15 (1973).

[13] K. Moriya et al. (CLAS collaboration), Phys. Rev. C 87, 035206 (2013).

[14] G. Agakishiev et al. (HADES collaboration), arXiv:1208.0205v2 (2013).

[15] R. Roosen et al., Nuov. Cim. A 49 2, 217 (1979). 